\title{
Molecular Targeting of ERKs/RSK2 Signaling Axis in Cancer Prevention
}

\author{
Sun-Mi Yoo ${ }^{1}$, Sung Jun $\mathrm{Cho}^{2}$, Yong-Yeon Cho ${ }^{1}$ \\ ${ }^{1}$ College of Pharmacy, The Catholic University of Korea, Bucheon, Korea, ${ }^{2}$ University Honors Program, Twin Cities College of Liberal Arts, University \\ of Minnesota, Minneapolis, MN, USA
}

\begin{abstract}
RSK2 is a downstream signaling protein of ERK1 and ERK2 and plays a key role in physiological homeostasis. For this reason, RSK2 is a highly conserved protein among the p90RSK family members. In its location in the signaling pathway, RSK2 is a kinase just upstream of transcription and epigenetic factors, and a few kinases involved in cell cycle regulation and protein synthesis. Moreover, activation of RSK2 by growth factors is directly involved in cell proliferation, anchorage-independent cell transformation and cancer development. Direct evidences regarding the etiological roles of RSK2 in cancer development in humans have been published by our research group illustrating that elevated total- and phospho-RSK2 protein levels mediated by ERK1 and ERK2 are higher in skin cancer tissues compared to normal skin tissues. Notably, it has been shown that RSK2 ectopic expression in JB6 Cl41 cells induces cell proliferation and anchorageindependent cell transformation. Importantly, knockdown of RSK2 suppresses Ras-mediated foci formation and anchorage-independent colony growth of cancer cells. Kaempferol is a one of the natural compounds showing selectivity in inhibiting RSK2 activity in epidermal growth factor-induced G1/S cell cycle transition and cell transformation. Thus, ERKS/RSK2 signaling axis is an important target signaling molecule in chemoprevention.
\end{abstract}

(J Cancer Prev 2015;20:165-171)

Key Words: Carcinogenesis, Neoplastic cell transformation, Molecular targeting, ERKs/RSK2 signaling, Natural compounds

\section{INTRODUCTION}

Protein phosphorylation, a major regulatory mechanism of enzyme activity and stability, is a process of post-translational modification by addition of phosphate group into amino acid residues such as serine, threonine and tyrosine. ${ }^{1}$ The phosphorylation of proteins is generally induced by cellular perturbations such as stress stimuli and cancerous transformation. Growth factors such as epidermal growth factor (EGF) and fibroblast growth factor, environmental stresses such as ultraviolet irradiation, and cytokines induce the phosphorylation of diverse proteins depending on the cellular context and the specific signaling pathway. ${ }^{2}$ One of the well-known signaling pathways is the mitogen-activated protein kinases (MAPK) signaling pathway, which includes extracellular signal-regulated kinases (ERKs), Jun-N-terminal kinases (JNKs) and p38 kinases (p38 MAPKs) (Fig. 1). ${ }^{3}$ The $90 \mathrm{kDa}$ ribosomal S6 kinases (p90RSKs: RSKs) superfamily, RSK1, RSK2, RSK3, RSK4, MSK1, and MSK2, is composed of RSKs and mitogen- and stress-activated protein kinases (MSKs) subfamilies, which are categorized together by amino acid similarity and structure. ${ }^{5}$ The amino acid sequence and structure of p90RSKs are also distinguishable from $70 \mathrm{kDa}$ RSK (p70S6K). ${ }^{6}$ One of the striking features is that the RSK family of proteins contains two functional kinase domains, N-terminal kinase that phosphorylates the substrates and C-terminal kinase that stimulates the activation of RSK in one polypeptide. ${ }^{7}$ The RSK family members shares very high amino acid similarity, about $80 \%$ among RSK isotypes including RSK1, RSK2, RSK3, and RSK4

Received June 19, 2015, Revised July 11, 2015, Accepted July 13, 2015

Correspondence to: Yong-Yeon Cho

College of Pharmacy, The Catholic University of Korea, 43 Jibong-ro, Wonmi-gu, Bucheon 14662, Korea

Tel: +82-2-2164-4092, Fax: +82-2-2164-4059, E-mail: yongyeong@catholic.ac.kr, ORCID: Yong-Yeon Cho, http://orcid.org/0000-0003-1107-2651

Copyright (C) 2015 Korean Society of Cancer Prevention

(c) This is an Open Access article distributed under the terms of the Creative Commons Attribution Non-Commercial License (http://creativecommons.org/licenses/by-nc/4.0) which permits unrestricted non-commercial use, distribution, and reproduction in any medium, provided the original work is properly cited. 


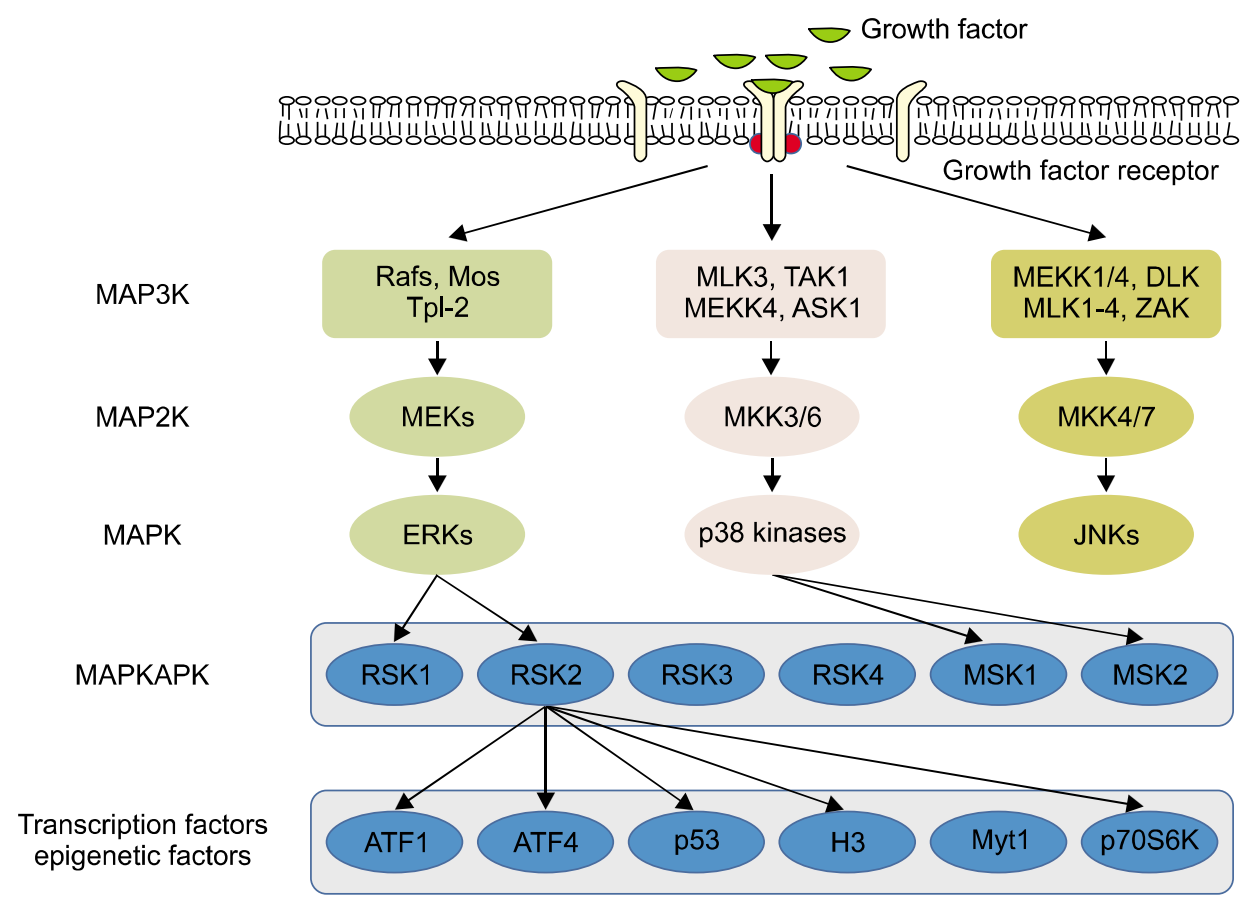

Figure 1. Growth factor-mediated signaling pathways. Growth factors such as epidermal growth factor bind to specific receptor on the cytoplasmic membrane and induce dimerization. The receptors induce autophosphorylation at tyrosine residues in cytoplasmic surface and transduce its activation signaling to many transcription factors through MAP kinase signaling pathways, which include extracellular-signal-regulated kinases (ERKs), p38 kinases (p38 MAPKs) and Jun-N-terminal kinases (JNKs). Currently, p90RSK superfamily is located in the downstream kinase of ERKs and p38 MAPKs and upstream of transcription factors, epigenetic factors and some of kinases regulating cell cycle distribution and protein synthesis. MAPKs, mitogen-activated protein kinases; Tpl-2, tumor promotion locus 2 (known as MAP3K8); MLK, mixed-lineage kinase; TAK, TGF $\beta$-activated kinase; ASK, apoptosis signal-regulating kinase; DLK, dual leucine zipper kinase (known as MAP3K12); ZAK, sterile alpha motif and leucine zipper containing kinase; ATF1, activating transcription factor 1; Myt1, myelin transcription factor 1 .

and about $63 \%$ between MSK1 and MSK2 in humans. ${ }^{5}$ Interspecies homology of RSKs between human and mouse also showed over $95 \%$ amino acid similarity, indicating that amino acid sequence of RSKs is well-conserved and plays a specific key role in maintaining homeostasis in physiological context. One example regarding the specific distinguishing roles among RSK isotypes is well identified in the Coffin-Lowry Syndrome (CLS), which is caused by the loss-of-function of RSK2, but not by other RSK isotypes including RSK1, RSK3, RSK4, MSK1, and MSK2. ${ }^{7}$ This indicates that RSK2 physiological function is not redundant with other RSKs isotypes. ${ }^{8}$ Notably, RSK2 expression is highly observed in different cancer cell lines ${ }^{9}$ and solid cancers compared to premalignant cells and normal tissues. ${ }^{9.10}$ The RSK2 activation is regulated by the upstream kinases including ERK1 and ERK2. ${ }^{10.11}$ Thus, RSK2 plays a key role in cell transformation and cancer development in humans. In this review, we will discuss the role of ERKs/RSK2 signaling on cell transformation and cancer development, and the importance of molecular targeting on the ERKs/RSK2 signaling axis for chemoprevention and cancer therapy.

\section{MAIN SUBJECT}

\section{Tumor promoter signaling}

MAPKs are Ser/Thr kinases that convert extracellular stimuli into a wide range of cellular responses including gene expression, mitosis, metabolism, motility, survival, apoptosis and differentiation. Binding of growth factors to the specific receptors induces dimerization and activation of receptor tyrosine kinases in cytoplasmic membrane, resulting in induction of autophosphorylation at tyrosine residue(s). ${ }^{12}$ These phosphorylation(s) serve as binding sites for specific proteins that contain Src homology 2 or phosphotyrosine-binding domains, such as growth factor receptor-binding protein-2 (Grb2). ${ }^{13}$ As a complex between Grb2 and Ras is formed, son of sevenless (SOS) is recruited from the cytoplasm to the membrane and stimulates the GDP exchange to Ras by GTP that is essential for a positive regulation of Ras activity. ${ }^{14}$ The Ras containing GTP interacts 
directly with its downstream target effector proteins such as Raf proteins, ${ }^{15}$ resulting in activation of Raf kinases. ${ }^{16}$ Activated Rafs bind and phosphorylate the dual specificity kinases, MEK1/2, which in turn, phosphorylate ERK1/2 at a conserved Thr-Glu-Tyr motif. ${ }^{17-19}$ Eventually, activated ERK1/2 bind at the ERK docking motif in C-terminal domain (CTD) of RSKs and phosphorylate the linker domain (LD) and C-terminal kinase domain (CTKD) of RSKs, resulting in the activation of N-terminal kinase activity. ${ }^{20}$

The importance of Ras/Raf/MEKs/ERKs/RSKs signaling pathway is emphasized by many of human solid tumors containing constitutive active mutations in Ras and/or Raf with a high percentage in pancreatic, colon, breast, ovarian, prostate, lung, melanoma and other cancers. ${ }^{21}$ The gain-of-function mutation of Ras or Raf transduces their activation signal to downstream kinases through a phospho-conveyer system even without stimulation of upstream activation signals initiated by interaction of growth factors and specific receptors. Based on these reasons, many scientists have tried to identify or synthesize specific small molecules targeting Ras and Raf proteins. When cells were stimulated with tumor promoters such as EGF or 12-Otetradecanoylphorbol13-acetate (TPA), phosphorylation and activation of MAPK kinase signaling molecules were frequently observed. ${ }^{20,22}$ Because the phosphorylation signaling is activated within a few minutes after tumor promoter stimulation, MAPKs belong to immediate early response genes. ${ }^{23}$ However, increasing evidences have demonstrated that there are functional differences between isotypes of MAPKs. For example, strong or sustained activation of Raf signal induces a G1-specific cell cycle arrest through induction of p21, resulting in inhibition of cyclin-D- and -E-dependent kinases. ${ }^{24,25}$ However, moderate Raf activity is sufficient to induce cyclin D expression and DNA synthesis. ${ }^{24}$ These results suggest that there are complicated feedback regulations of proteins that modulate activation of signaling pathways. One of the extensively studied negative feedback loops is the negative feedback inhibition of Raf activity by AKT and serum/glucocorticoid inducible kinase. ${ }^{26,27}$ We have reported a critical clue that the interface between ERK1/2 and RSK2 activity may contain an negative feedback loop because RSK2 deficiency increases the total protein levels of ERK1 and ERK2. ${ }^{28}$ Moreover, when cells are stimulated with growth factors such as EGF, the susceptibility of ERK1 and ERK2 phosphorylation is dramatically enhanced by RSK2 deficiency. ${ }^{28}$ In contrast to G1 specific arrest by the Raf/MEKs/ERKs signaling pathway, ${ }^{25}$ most tumors have increased and sustained activation of the Raf/MEKs/ ERKs pathway. ${ }^{21}$ Our research group has provided evidences that tumor promoter stimulation induces the phosphorylation of MEKs and ERKs. ${ }^{22}$ Recent evidence highlights the importance of
RSK2 in cell proliferation and transformation. RSK2 deficiency attenuates cell proliferation in mouse embryonic fibroblasts (MEFs) compared with RSK2 wildtype MEFs. ${ }^{22}$ The potential of RSK2 as an oncokinase was suggested by the fact that ectopic expression of RSK2, which enhanced anchorage-independent cell transformation without EGF stimulation in JB6 Cl41 cells. ${ }^{22}$ Interestingly, colony formation in the RSK2 expressing JB6 Cl41 cells in soft agar without EGF stimulation was increased by stimulation of EGF, indicating that overexpressed RSK2 activity was also enhanced by EGF stimulation. Moreover, proapoptotic BAD inactivation was achieved through RSK2 by activation of ERKs. ${ }^{29}$ Thus, ERKs/RSK2 signaling axis plays an important role in signaling node for the tumor promoter-induced cell proliferation and transformation.

\section{ERK1/2-mediated RSK2 activation}

The RSK2 activation mechanisms are very complicated due to the diverse nature of RSKs, the consecutive activity regulation by phosphorylation, the agonist-specific temporal activity regulation, the changing spatial distribution in cellular organelles, the existence of diverse interacting proteins, and the possession of two unique kinase domains in a polypeptide. ${ }^{5}$ The N-terminal kinase domain (NTKD) is classified as a AGC group kinase family (which includes PKA, PKG, and PKC), and the CTKD belongs to the calcium/calmodulin-dependent (CaMK) kinase family. ${ }^{20}$ The CTD of RSK2 contains a non-canonical ERK docking signal, Leu-X-X-Lys/ Arg-Lys/Arg-X-X-X-X-X-Leu, which is different from classical D-type ERK1/2 docking domains. ${ }^{17}$ Moreover, a similar motif has been found in several other MAPK substrates including PTP-SL, a protein tyrosine phosphatase, ${ }^{19}$ phosphodiesterase 4D, cAMP-specific phosphoesterase. ${ }^{18}$ Although the CTD of RSK2 is not typical of the best-characterized consensus sequences for D-type ERK1/2 docking domains and appears to fit the kinase interaction motif consensus sequences, ${ }^{4,18}$ an in vitro kinase assay demonstrated that RSK2 was activated by ERK1 and ERK2, not by p38 kinases. ${ }^{9}$ Generally, interaction of ERK1 or ERK2 with RSK2 has high affinity through $\mathrm{D}$ domain. The investigation of the interaction between ERK1/2 and RSK1 indicated that inactive ERK1/2 has a higher affinity with RSK1 than active forms of ERK1/2 following mitogen stimulation. ${ }^{30}$ Additionally, autophosphorylation of a serine residue near ERK1/2 docking domain of RSK1 promotes its dissociation from ERK1/2. ${ }^{30}$ Based on these evidences, a possible RSK2 activation mechanism is the activation of inactive ERK1 or ERK2 with RSK2 pre-complex by growth factor stimulation, which results in dissociation of ERK1/2 from CTD of RSK2. Activated ERK1 or ERK2 induces phosphorylation of RSK2 at Thr365/Ser369 
located in the LD and at Thr577 located in the CTKD, resulting in the induction of RSK2 CTKD kinase activity. The activated CTKD phosphorylates another serine residue (Ser386) in the LD, which serves as a PDK1 docking motif. The PDK1 docking at the LD domain of RSK2 facilitates phosphorylation of Ser227 in NTKD. Thus, the CTKD of RSK2 is essential for the initiation of RSK2 activation process, resulting in activation of RSK2 NTKD. The cascade kinase reaction indicated that the kinase domain, NTKD of RSK2, played a key role in substrate phosphorylation. ${ }^{9}$ The RSK2 protein purified from Escherichia coli did not have the ability to phosphorylate nuclear factor of activated T-cells (NFAT3)-261-365 protein, which is known to be the best substrate of RSK2 having about $\mathrm{Km}=0.3559 \mu \mathrm{M}$. $^{31}$ In contrast, when the RSK2 proteins were activated by active ERK2, RSK2 recovers the ability to phosphorylate NFAT3-261-365. ${ }^{9}$ Interestingly, RSK2 proteins not containing either NTKD or CTKD totally lost the ability to phosphorylate NFAT3-261-365 proteins, ${ }^{9}$ indicating that CTKD activation of RSK2 is indispensable to activate NTKD of RSK2.

\section{ERK1/2-mediated RSK2 signaling in cell transfor- mation}

Up-regulation of the MAPK signaling pathway promotes cell proliferation and enhances cell survival in various cancer cells. ${ }^{21}$ When cells are stimulated with a survival growth factor, such as brain-derived neurotropic factor, RSK2 induces phosphorylation of proapoptotic BAD protein, ${ }^{32}$ resulting in enhancement of cell survival. Our research group found that the stimulation of tumor promoters, such as EGF or TPA, induces the phosphorylation of ERK1/2 and RSKs, resulting in induction of G1/S cell cycle transition and cell proliferation. ${ }^{22}$ These results were supported by experiments, which showed that RSK2 deficiency attenuates cell proliferation compared to $\mathrm{RSK}^{+/+} \mathrm{MEFs}^{22}$ Recently, when cells were irradiated with ultraviolet light, RSK2 induced glycogen synthase kinase $3 \beta$ (GSK3 $\beta$ ) phosphorylation at Ser9. ${ }^{28}$ Since activation of GSK3 $\beta$ (non-phosphorylated GSK3 $\beta$ at Ser9) induces cell cycle arrest and apoptosis, $\mathrm{RSK}^{+/+}$MEFs showed resistance to apoptosis by ultraviolet irradiation. ${ }^{28}$ These results indicate that ERKs-mediated RSK2 signaling pathway induces not only cell proliferation but also cell survival. In this signaling pathway, RSK2 activity was correlated with cell transformation. When RSK2 was introduced to cells using an ectopic expression vector, the cells showed increased anchorage-independent colony formation without EGF stimulation. ${ }^{22}$ Furthermore, critical evidences highlighted the importance of Ras/MEKs/ERKs/RSK2 signaling pathway in cell transformation. For example, The Ras/MEKs/ERKs signaling axis-mediated RSK2 activation is proven by the knockdown of RSK2 with si-RNA RSK2 in cells stably expressing constitutively active Ras (CA-Ras) alone or CA-Ras and RSK2. ${ }^{22}$ The results showed that RSK2 knockdown suppressed foci formation in NIH3T3 cells. ${ }^{22}$ The RSK2 total protein profile indicates that RSK2 protein levels are higher in cancer cells than that of nonmalignant cells. ${ }^{9}$ Importantly, kaempferol, a natural compound harboring RSK2 selective inhibitory effect, inhibits cell proliferation in a dose dependent manner. ${ }^{9}$ The etiological evidence that RSK2 is involved in cancer development in humans was provided by skin cancer tissue array. Immunohistofluorescence array containing 70 core human skin cancer tissues and 10 normal skin tissues demonstrated that total RSK2 protein levels were higher in skin cancer tissues than that of normal tissues. ${ }^{9}$ Moreover, activated RSK2 protein, phospho-RSK2 at Thr577, was elevated in skin cancer tissues compared to normal skin tissues. ${ }^{10}$ Elevated total- and phospho-RSK2 protein levels were increased in sub-categorized human skin cancer tissues, such as squamous cell carcinoma, basal cell carcinoma and malignant melanoma, compared to normal skin tissues. ${ }^{10}$ Importantly, HaCaT cells, a premalignant human skin keratinocyte cell line, N/TERT-1 cells, a human skin keratinocyte cell line immortalized by telomerase, ${ }^{33} \mathrm{SCC}-13$ cells, a human skin epidermal squamous cell carcinoma cell line ${ }^{34}$ and SK-MEL-28 malignant melanoma cells contain different levels of endogenous RSK2 protein, and RSK2 knockdown effects on the cell proliferation were correlated with endogenous RSK2 protein levels. ${ }^{10}$ Based on these results, it can be concluded that ERK1/2mediated RSK2 protein activation plays a key role in cell survival and cell proliferation, resulting in cancer development in humans.

\section{Molecular Targeting of ERKs/RSK2 in chemopre- vention}

ERKs are one of the more well-known MAPK signaling molecules that are located of downstream of cell surface receptors and other cytoplasmic signaling proteins whose functions are deregulated in cancer and other human pathogenic disorders. ${ }^{3}$ Due to their importance and involvement in cell proliferation and survival, ERK1 and ERK2 have attracted intense research interest to identify small molecules that inhibit ERK1 and ERK2 activities. The rationale is supported by the mutational activation and/or overexpression of upstream signaling molecules that activate the ERKs. Our results also demonstrated that growth factors and environmental stresses induce the phosphorylation of ERK1 and ERK2 in a short time after treatment. ${ }^{10.22}$ To date, research for the identification and development of small molecules that target the Ras/Rafs/MEKs/ERKs/RSK2 signaling axis have been focused on upstream proteins of ERKs and RSK2. Although, the Raf/MEKs/ 


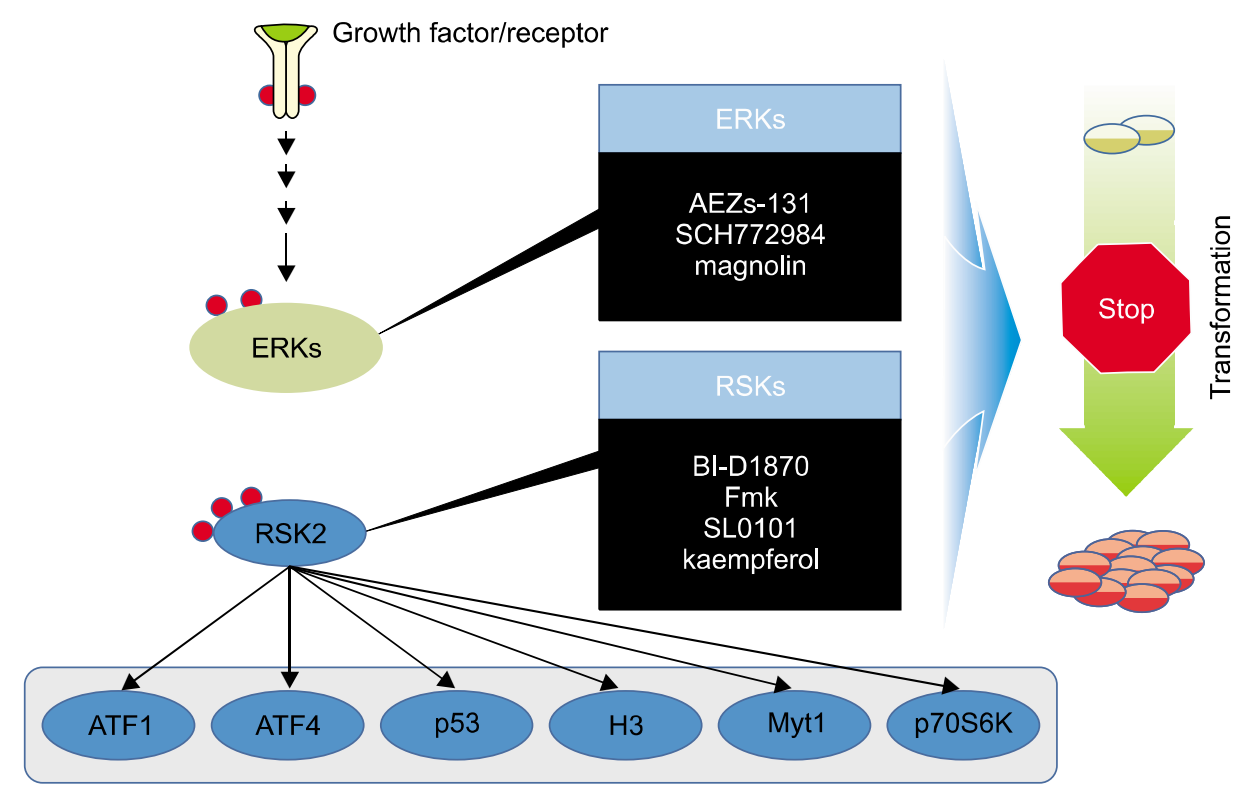

Figure 2. Inhibitors targeting extracellular signal-regulated kinases (ERKs) and RSK2. Chemical compounds, AEZs-131, SCH772984, and magnolin, targeting ERKs activity are synthesized or identified. Magnolin, a natural compound abundantly found in Shin-Yi, inhibits cell proliferation and anchorage-independent transformation induced by tumor promoters such as EGF. BI-D1870 is a derivative frompyrido[2,3-d]pyridimidine group of Src inhibitors targeting N-terminal kinase domain of RSK2. Fmk is an irreversible C-terminal kinase inhibitor of RSK2 by covalent bond formation at Cys436. SL0101, kaempferol-3-O-(3",4"-di-O-acetyl- $\alpha$-L-rhamnopyranoside), is a natural compound abundantly found in Forsteronia refracta, which found in the South America Amazon rainforest, and have inhibitory effects of RSKs by targeting to N-terminal kinase domain. Kaempferol, a natural compound found in dietary foods such as leafs of green onion, targeting N-terminal kinase domain of RSK2, but not RSK1 and RSK3. Kaempferol also inhibits cell proliferation and anchorage-independent cell transformation. ATF1, activating transcription factor 1; Myt1, myelin transcription factor 1.

ERKs cascade is a critical signaling axis of Ras-mediated carcinogenesis, recent studies have clearly demonstrated that Ras is involved in the activation of effector signaling molecules. ${ }^{35}$ For example, the $\mathrm{p} 110$ catalytic subunits ( $\mathrm{p} 110 \alpha, \beta, \gamma$, and $\delta$ ) of class I phosphatidylinositol 3-kinases, the Ral small GTPase specific GEFs (RalGDS, Rgl, Rgl2, and Rgl3), the Tiam1 Rac small GTPasespecific GEF and the phospholipase $C$ epsilon plays important roles in Ras-induced oncogenesis. ${ }^{35.36}$ This suggests that aberrant inhibition of Ras and Ras/Raf/MEKs by small molecules may express unwanted effects. Therefore, we have considered that targeting of signaling molecules closely located to transcription factors in signaling cascades may promise more specific effects against target diseases with fewer side effects (Fig. 2). BI-D1870, a derivative frompyrido[2,3-d]pyridimidine group of Src inhibitors, is a cell permeable inhibitor with relative specificity for the RSK family with about 10 to $30 \mathrm{nM}$ of $\mathrm{IC}_{50}$ value. ${ }^{37,38}$ The results demonstrated that BI-D1870 inhibited the EGF- or TPA-mediated phosphorylation of GSK3 $\beta$ and LKB1 by targeting of NTKD of RSKs. $^{37}$ A different type of RSK2 inhibitor, pyrrolopyrimidine fmk, was developed. ${ }^{39}$ The fmk targeted CTKD of RSK2 and inhibited RSK2 CTKD activity with $15 \mathrm{nM}$ of $\mathrm{IC}_{50}$ and $200 \mathrm{nM}$ of the half maximal effective concentration $\left(\mathrm{EC}_{50}\right){ }^{39}$ The compound inhibits irreversible C-terminal kinase activity of RSK2 by covalent addition of chloromethylketone to thiol group of Cys436. ${ }^{39}$ Recently, our research group found that kaempferol, a natural compound abundantly found in editable dietary plants, targeted the N-terminal kinase activity by binding to the active pocket of RSK2. ${ }^{9}$ The docking score of kaempoferol on the active pocket of RSK2 NTKD is about $-12.8 \mathrm{kcal} / \mathrm{mol}$ which is similar to the SL0101 (kaempferol-3-O-(3",4"-di-O-acetyl- $\alpha$-L-rhamnopyranoside) docking score which is about $-13.3 \mathrm{kcal} / \mathrm{mol}$. ${ }^{9.40}$ Our computational model suggested that kaempferol formed three hydrogen bonds with the backbone atoms of $\mathrm{Asp}^{148}$, Leu ${ }^{150}$, and Leu ${ }^{74}$ or Lys ${ }^{100} \cdot 9$ Moreover, heterocyclic ring system of kaempferol lies in the hydrophobic region of the RSK2 NTKD catalytic site where it is occupied by ATP. ${ }^{9}$ More recently, X-ray crystal structure of RSK2 NTKD and kaempferol reveals that amino acids including Asp ${ }^{148}$, $\mathrm{Glu}^{197}$, and Lys ${ }^{100}$ participate in specific interactions between the inhibitor and the protein moiety of RSK2 NTKD to form specific hydrogen bonds. ${ }^{41}$ Interestingly, $\mathrm{Ile}^{50}, \mathrm{Ile}^{52}, \mathrm{Phe}^{79}, \mathrm{Leu}^{102}, \mathrm{Val}^{131}$, Leu $^{147}$, Leu ${ }^{150}$, Leu ${ }^{155}$, Leu $^{200}$, and Phe ${ }^{212}$ were shown to participate in the interaction between SL0101 and RSK2 N-terminal domain and NTKD. ${ }^{41}$ Our research group found that kaempferol inhibited not only RSK2 N-terminal kinase activity with a $\mathrm{IC}_{50}$ value of to 
about $7 \mu \mathrm{M}$ in vitro but also cell proliferation in cell culture with a IC 50 value of to about $30 \mu \mathrm{M} .{ }^{9.22}$ Although, the $\mathrm{IC}_{50}$ values of SL0101 in the RSK2 NTKD activities were different (approximately from $89 \mathrm{nM}$ to $7 \mu \mathrm{M}$ ) from kaempferol in vitro, ${ }^{41,42}$ the $\mathrm{IC}_{50}$ values on cell proliferation were similar in both SL0101 and kaempferol, approximately from 20 to $40 \mu \mathrm{M}$, in many different premalignant and cancer cells. ${ }^{92,42,43}$ However, accumulating evidences indicate that the sugar side chains in natural compounds are cleaved in the intestinal flora to increase the intestinal absorption of those particular compounds. ${ }^{44.45}$ Importantly, after oral administration, kaempferol was detected in human plasma, about a concentration of $800 \mathrm{nmol} / \mathrm{L}$, and urine ${ }^{46}$ indicating that kaempferol is absorbed in the intestine. Thus, we believe similar concentration of SL0101 and kaempferol show similar effects on the cell proliferation.

\section{FURTHER DIRECTIONS}

Current studies on the p90RSK family are mainly focused on RSK2 because RSK2 mutation and genomic deletion causes a human genetic disease known as CLS. For the last decade, the research on the etiological role of RSK2 in human cancers have been gradually accelerating, and now research scientists believe that RSK2 is a key signaling molecule and playing an important role in cell proliferation and transformation. Based on these efforts, some research groups have found RSK inhibitors including ones that inhibit RSK2. One of the representative natural compounds having inhibitory effects on the RSK2 activity is SL0101, extracted from Forsteronia refracta, which found in the South America Amazon rainforest, and kaempferol, abundantly found in editable dietary plants such as leafs of green onion and endives. Although, SL0101 showed lower $\mathrm{IC}_{50}$ values than that of kaempferol in vitro assay using holoenzymes, the effective IC $_{50}$ concentration of kaempferol is similar to that of SL0101 on cell proliferation and transformation. Although kaempferol is not suitable to be used in cancer therapy directly, kaempferol might be potentiated as a chemopreventive agent because of its distribution in editable plants. Moreover, kaempferol may use as a mother molecule to develop improved synthetic chemical compounds having higher selectivity and efficacy against RSK2.

\section{ACKNOWLEDGMENTS}

This work was supported in part by the Ministry of Science, ICT and Future Planning (NRF-2012M3A9B6055466 and -2014R1A 2A1A11050004).

\section{CONFLICTS OF INTEREST}

No potential conflicts of interest were disclosed.

\section{REFERENCES}

1. Friedmann DR, Marmorstein R. Structure and mechanism of non-histone protein acetyltransferase enzymes. FEBS J 2013;280: 5570-81.

2. Manna PR, Stocco DM. The role of specific mitogen-activated protein kinase signaling cascades in the regulation of steroidogenesis. J Signal Transduct 2011;2011:821615.

3. Roberts PJ, Der CJ. Targeting the Raf-MEK-ERK mitogen-activated protein kinase cascade for the treatment of cancer. Oncogene 2007;26:3291-310.

4. Romeo Y, Zhang X, Roux PP. Regulation and function of the RSK family of protein kinases. Biochem J 2012;441:553-69.

5. Arul N, Cho YY. A rising cancer prevention target of RSK2 in human skin cancer. Front Oncol 2013;3:201.

6. Dennis PB, Pullen N, Pearson RB, Kozma SC, Thomas G. Phosphorylation sites in the autoinhibitory domain participate in p70(s6k) activation loop phosphorylation. J Biol Chem 1998;273: 14845-52.

7. Moller DE, Xia CH, Tang W, Zhu AX, Jakubowski M. Human rsk isoforms: cloning and characterization of tissue-specific expression. Am J Physiol 1994;266:C351-9.

8. Cho YY, He Z, Zhang Y, Choi HS, Zhu F, Choi BY, et al. The p53 protein is a novel substrate of ribosomal S6 kinase 2 and a critical intermediary for ribosomal S6 kinase 2 and histone $\mathrm{H} 3$ interaction. Cancer Res 2005;65:3596-603.

9. Cho YY, Yao K, Pugliese A, Malakhova ML, Bode AM, Dong Z. A regulatory mechanism for RSK2 $\mathrm{NH}(2)$-terminal kinase activity. Cancer Res 2009;69:4398-406.

10. Cho YY, Lee MH, Lee CJ, Yao K, Lee HS, Bode AM, et al. RSK2 as a key regulator in human skin cancer. Carcinogenesis 2012;33: 2529-37.

11. Gavin AC, Ni Ainle A, Chierici E, Jones M, Nebreda AR. A p90(rsk) mutant constitutively interacting with MAP kinase uncouples MAP kinase from p34(cdc2)/cyclin B activation in Xenopus oocytes. Mol Biol Cell 1999;10:2971-86.

12. Lane MD, Slieker LJ, Olson TS, Martensen TM. Post-translational acquisition of ligand binding- and tyrosine kinase-domain function by the epidermal growth factor and insulin receptors. J Recept Res 1987;7:321-54.

13. Sakaguchi K, Okabayashi Y, Kido Y, Kimura S, Matsumura Y, Inushima $\mathrm{K}$, et al. Shc phosphotyrosine-binding domain dominantly interacts with epidermal growth factor receptors and mediates Ras activation in intact cells. Mol Endocrinol 1998;12: 536-43.

14. McLaughlin S, Aderem A. The myristoyl-electrostatic switch: a modulator of reversible protein-membrane interactions. Trends Biochem Sci 1995;20:272-6.

15. Kolch W, Heidecker G, Lloyd P, Rapp UR. Raf-1 protein kinase is required for growth of induced NIH/3T3 cells. Nature 1991;349: 426-8.

16. Troppmair J, Bruder JT, App H, Cai H, Liptak L, Szeberényi J, et al. Ras controls coupling of growth factor receptors and protein kin- 
ase $C$ in the membrane to Raf- 1 and B-Raf protein serine kinases in the cytosol. Oncogene 1992;7:1867-73.

17. Tanoue T, Nishida E. Molecular recognitions in the MAP kinase cascades. Cell Signal 2003:15:455-62.

18. MacKenzie SJ, Baillie GS, McPhee I, Bolger GB, Houslay MD. ERK2 mitogen-activated protein kinase binding, phosphorylation, and regulation of the PDE4D cAMP-specific phosphodiesterases. The involvement of $\mathrm{COOH}$-terminal docking sites and $\mathrm{NH2}$-terminal UCR regions. J Biol Chem 2000;275:16609-17.

19. Pulido R, Zúñiga A, Ullrich A. PTP-SL and STEP protein tyrosine phosphatases regulate the activation of the extracellular signal-regulated kinases ERK1 and ERK2 by association through a kinase interaction motif. EMBO J 1998;17:7337-50.

20. Frödin M, Gammeltoft S. Role and regulation of $90 \mathrm{kDa}$ ribosomal S6 kinase (RSK) in signal transduction. Mol Cell Endocrinol 1999; 151:65-77.

21. Sebolt-Leopold JS, Herrera R. Targeting the mitogen-activated protein kinase cascade to treat cancer. Nat Rev Cancer 2004:4:937-47.

22. Cho YY, Yao K, Kim HG, Kang BS, Zheng D, Bode AM, et al. Ribosomal S6 kinase 2 is a key regulator in tumor promoter induced cell transformation. Cancer Res 2007;67:8104-12.

23. Dziema H, Oatis B, Butcher GQ, Yates R, Hoyt KR, Obrietan K. The ERK/MAP kinase pathway couples light to immediate-early gene expression in the suprachiasmatic nucleus. Eur J Neurosci 2003;17:1617-27.

24. Sewing A, Wiseman B, Lloyd AC, Land H. High-intensity Raf signal causes cell cycle arrest mediated by p21Cip1. Mol Cell Biol 1997; 17:5588-97.

25. Pumiglia KM, Decker SJ. Cell cycle arrest mediated by the MEK/mitogen-activated protein kinase pathway. Proc Natl Acad Sci U S A 1997;94:448-52.

26. Guan KL, Figueroa C, Brtva TR, Zhu T, Taylor J, Barber TD, et al. Negative regulation of the serine/threonine kinase B-Raf by Akt. J Biol Chem 2000;275:27354-9.

27. Zhang BH, Tang ED, Zhu T, Greenberg ME, Vojtek AB, Guan KL. Serum- and glucocorticoid-inducible kinase SGK phosphorylates and negatively regulates B-Raf. J Biol Chem 2001;276:31620-6.

28. Lee CJ, Lee MH, Lee JY, Song JH, Lee HS, Cho YY. RSK2-induced stress tolerance enhances cell survival signals mediated by inhibition of GSK3 $\beta$ activity. Biochem Biophys Res Commun 2013:440:112-8.

29. Shimamura A, Ballif BA, Richards SA, Blenis J. Rsk1 mediates a MEK-MAP kinase cell survival signal. Curr Biol 2000;10:127-35.

30. Roux PP, Richards SA, Blenis J. Phosphorylation of $\mathrm{p} 90$ ribosomal S6 kinase (RSK) regulates extracellular signal-regulated kinase docking and RSK activity. Mol Cell Biol 2003;23:4796-804.

31. Cho YY, Yao K, Bode AM, Bergen HR 3rd, Madden BJ, Oh SM, et al. RSK2 mediates muscle cell differentiation through regulation of NFAT3. J Biol Chem 2007;282:8380-92.

32. Bonni A, Brunet A, West AE, Datta SR, Takasu MA, Greenberg ME. Cell survival promoted by the Ras-MAPK signaling pathway by transcription-dependent and -independent mechanisms. Science
1999:286:1358-62.

33. Dickson MA, Hahn WC, Ino Y, Ronfard V, Wu JY, Weinberg RA, et al. Human keratinocytes that express hTERT and also bypass a p16(INK4a)-enforced mechanism that limits life span become immortal yet retain normal growth and differentiation characteristics. Mol Cell Biol 2000;20:1436-47.

34. Rubin AL, Parenteau NL, Rice RH. Coordination of keratinocyte programming in human SCC-13 squamous carcinoma and normal epidermal cells. J Cell Physiol 1989;138:208-14.

35. Repasky GA, Chenette EJ, Der CJ. Renewing the conspiracy theory debate: does Raf function alone to mediate Ras oncogenesis? Trends Cell Biol 2004;14:639-47.

36. Shaw RJ, Cantley LC. Ras, PI(3)K and mTOR signalling controls tumour cell growth. Nature 2006:441:424-30.

37. Sapkota GP, Cummings L, Newell FS, Armstrong C, Bain J, Frodin $\mathrm{M}$, et al. BI-D1870 is a specific inhibitor of the p90 RSK (ribosomal S6 kinase) isoforms in vitro and in vivo. Biochem J 2007:401:29-38.

38. Bain J, Plater L, Elliott M, Shpiro N, Hastie CJ, McLauchlan H, et al. The selectivity of protein kinase inhibitors: a further update. Biochem J 2007:408:297-315.

39. Cohen MS, Zhang C, Shokat KM, Taunton J. Structural bioinformatics-based design of selective, irreversible kinase inhibitors. Science 2005:308:1318-21.

40. Nguyen TL, Gussio R, Smith JA, Lannigan DA, Hecht SM, Scudiero DA, et al. Homology model of RSK2 N-terminal kinase domain, structure-based identification of novel RSK2 inhibitors, and preliminary common pharmacophore. Bioorg Med Chem 2006; 14:6097-105.

41. Utepbergenov D, Derewenda U, Olekhnovich N, Szukalska G, Banerjee B, Hilinski MK, et al. Insights into the inhibition of the p90 ribosomal S6 kinase (RSK) by the flavonol glycoside SL0101 from the $1.5 \AA$ crystal structure of the N-terminal domain of RSK2 with bound inhibitor. Biochemistry 2012;51:6499-510.

42. Smith JA, Poteet-Smith CE, Xu Y, Errington TM, Hecht SM, Lannigan DA. Identification of the first specific inhibitor of $\mathrm{p} 90$ ribosomal S6 kinase (RSK) reveals an unexpected role for RSK in cancer cell proliferation. Cancer Res 2005:65:1027-34.

43. Clark DE, Errington TM, Smith JA, Frierson HF Jr, Weber MJ, Lannigan DA. The serine/threonine protein kinase, p90 ribosomal S6 kinase, is an important regulator of prostate cancer cell proliferation. Cancer Res 2005:65:3108-16.

44. Day AJ, DuPont MS, Ridley S, Rhodes M, Rhodes MJ, Morgan MR, et al. Deglycosylation of flavonoid and isoflavonoid glycosides by human small intestine and liver beta-glucosidase activity. FEBS Lett 1998:436:71-5.

45. Walle T. Absorption and metabolism of flavonoids. Free Radic Biol Med 2004:36:829-37.

46. DuPont MS, Day AJ, Bennett RN, Mellon FA, Kroon PA. Absorption of kaempferol from endive, a source of kaempferol-3-glucuronide, in humans. Eur J Clin Nutr 2004:58:947-54. 